\title{
EXTRACCIÓN POR FLUIDO SUPERCRÍTICO DE CO, Y CUANTIFICACIÓN DE CAPSAICINOIDES DE ROCOTO (Capsicum Pubescen) DEL VALLE DEL MANTARO
}

\section{EXTRACTION BY CO2 SUPERCRITICAL FLUID AND HOT PEPPER CAPSAICINOIDS QUANTIFICATION (Capsicum Pubescen) FROM THE MANTARO VALLEY}

\section{Norma Gamarra Mendoza1, Ivan Bontemps Hernández, Edith Coronel Bonifacio, Silvia Velásquez Rodríguez y Servan Granza Muñoz \\ Laboratorio de Investigación en Biotecnología de Alimentos de la Facultad de Industrias Alimentarias - UNCP}

\section{RESUMEN}

Los cromatogramas obtenidos permitieron identificar y cuantificar las fracciones de capsaicinoides de la oleorresina de rocoto. El tratamiento $\mathrm{T} 2 \mathrm{a} 60^{\circ} \mathrm{C}$ y 200 bar, permitió la mayor extracción a diferencia de las otras condiciones, siendo Nordihidrocapsaicina $(\mathrm{NDHC})=770.51$ (17\%); Capsaicina (CAP) $=1471.22(33 \%)$ y Dihidrocapsaicina $(\mathrm{DHC})=2116.19(49 \%)$ haciendo un total de capsaicinoides de 4357.92 $\mathrm{ug} / \mathrm{g} \mathrm{ms,} \mathrm{y} \mathrm{con} \mathrm{una} \mathrm{pungencia} \mathrm{de} \mathrm{SHU=65368.76;}$ la relación de estas fracciones 1:0.69:0.36 señala que la dihidrocapsaicina se encuentra en mayor proporción en esta especie de Capsicum pubescen. En conclusión, los resultados de este estudio demostraron que el SFE - $\mathrm{CO}_{2}$ es un método disponible para extracciones rutinarias y selectivas de capsaicinoides de rocoto seco y molido con $12 \%$ de humedad y con muchas ventajas de fácil extracción, libre de contaminantes y cuantificación rápida de fracciones de capsaicinoides.

Palabras clave: capsaicinoids, supercritical-fluid - $\mathrm{CO}_{2}$, nordihidrocapsaicin, dihydrocapsaicin, capsaicin.

\section{ABSTRACT}

The chromatograms obtained allowed to identify and quantify the fractions of capsaicinoids of the oleoresin of Capsicum sp. (Hot peppers) T2 treatment at $40^{\circ} \mathrm{C}$ and 400 bar, permitted greater extraction as opposed to other conditions, being Nordihidrocapsaicin $(\mathrm{NDHC})=770.51$ $(17 \%)$, Capsaicina $(\mathrm{CAP})=1471.22(33 \%)$ and Dihidrocapsaicina $(\mathrm{DHC})=2116.19(49 \%)$ for a total of $4357.92 \mathrm{u} / \mathrm{g} \mathrm{ms}$ of capsaicinoids, and with a pungency of $\mathrm{SHU}=65368.76$, the relationship of these fractions indicates that the dihydrocapsaicin $1: 0.69: 0.36$ is in greater proportion in this species of Capsicum sp. In conclusion, the results of this study showed that the SFE-CO is an available method for routine extraction of capsaicinoids from dried and ground rocoto with $12 \%$ humidity and with many advantages of easy removal, free of contaminants, and rapid quantification of fractions of capsaicinoids.

Key words: capsaicinoids, supercritical-fluid - $\mathrm{CO}_{2}$, nordihidrocapsaicin, dihydrocapsaicin, capsaicin.

\section{INTRODUCCIÓN}

El género Capsicum, comúnmente denominado ají en el Perú, juega un rol importante en el sector hortícola del país. El rocoto o ají manzano perteneciente a la especie Capsicum pubescen es una de las especies de mayor cultivo después del paprika y aji escabeche y ampliamente utilizado en la gastronomía, su producción alcanzó a 14424.31 TM (Ministerio de Agricultura, 2005) y su comercialización se está posesionando en los mercados externos (China, USA, España, etc.). El Perú es uno de los centros

\footnotetext{
'nngamarram@hotmail.com
} 
de origen de la mayor cantidad de especies de Capsicum, que data desde la época preinca, esta diversidad biológica de nuestro país nos exige asumir una responsabilidad en el uso sostenible y conservación de estos recursos y la necesidad de prepararnos y exigirnos para aprovechar las nuevas tendencias del mercado.

El Consejo Internacional de Recursos Fitogenéticos con sede en Costa Rica (1979), reconoció a nivel mundial la importancia socioeconómica y científica de este género, considerado dentro de los recursos fitogenéticos de primera prioridad (Ortiz,1983). El género Capsicum presenta una gran oferta de especies y variedades, las cuales reúnen características de precocidad, productividad y demanda comercial que los convierte en un recurso genético promisorio para los sistemas productivos y hace viable su inclusión en agrocadenas de los mercados nacional e internacional. El ají por su contenido de componentes bioactivos, especialmente capsacinoides y carotenoides es de interés medicinal, farmacológico, alimentario, cosmecéutico cobra importancia para su investigación, mejoramiento de cultivo, producción, industrialización y comercialización. El potencial de compuestos naturales que ofrece el género Capsicum es impresionante, sin embargo en nuestro país muy poco se ha investigado para utilizar estas ventajas comparativas, competitivas, ya que las tendencias actuales a nivel mundial, consideran prioritarias la búsqueda de nuevos compuestos bioactivos, ingredientes, sabores, aromas naturales de matrices vegetales, para utilizarlos como alimentos saludables y nutritivos que orienten a mejores oportunidades de comercialización para posesionarse en mercados verdes de alimentos funcionales, nutracéuticos, cosmecéuticos, productos orgánicos e insumos exclusivos. Además de estos usos, los frutos se utilizan procesados 0 en fresco y bajo diferentes formas para la preparación y aliño de los alimentos. La calidad de los frutos del aji y de sus subproductos depende del color, el aroma y la pungencia (Kirschbaum et al., 2002a; y Macrae et al., 1993); particularmente el sabor pungente 0 picante originado por la presencia de capsaicinoides que son alcaloides que están presentes únicamente en el género capsicum y tienen propiedades medicinales y farmacológicas como anti cancerígenas, antiartríticas, antiinflamatorias (Howar et al 2000; Mazzone et al, 1999) neuropatías antidiabéticas, neurológicas - se le atribuye propiedades quimioterapéuticas (Kimet et al, 2004); sus aplicaciones en la industria alimentaria para la preparación de salsas pungentes; asimismo es usado en la agricultura como biopesticida, fungicida y en otros campos para preparar repelentes y bombas lacrimógenas, etc. Actualmente la extracción de los capsaicinoides de Capsicum, refieren a la extracción por fluido supercrítico de $\mathrm{CO}_{2}$, por la ventajas competitivas que ofrece, como es la selectividad, estabilidad, pureza, corto tiempo y proceso fácil. Asimismo el $\mathrm{CO}_{2}$ en condiciones supercríticas muestra una alta difusividad y alta solubilidad, comportándose como un excelente solvente. El Perú es uno de los centros de origen de la mayor cantidad de especies de Capsicum, que data desde la época del incanato, esta diversidad biológica de nuestro país nos exige asumir una responsabilidad en el uso sostenible y conservación de estos recursos y la necesidad de prepararnos y exigirnos para aprovechar las nuevas tendencias del mercado.

En el presente trabajo de investigación se realizó la extracción por fluido supecrítico de $\mathrm{CO}_{2}$ y la cuantificación por cromatografía líquida de alta eficiencia (HPLC) de capsaicinoides de ROCOTO (Capsicum pubescen), ya que a la fecha esta especie no ha recibido la atención científica ni tecnológica tanto botánica, genética, agronómica, fitoquímica, bioquímica etc., a pesar de que su cultivo en el valle del Mantaro es muy prospera y resistente al ataque de plagas; actualmente solamente crece a nivel de huertos 
familiares.

\section{MATERIAL Y MÉTODOS}

\section{Lugar de ejecución}

Laboratorio de investigación en biotecnología de alimentos de la UNCP - FAllA

Laboratorio de Ingeniería de Alimentos de la Universidad Peruana Unión de Lima.

\section{Materiales}

\section{Material biológico}

Género: Capsicum

Especie: Capsicum pubescen

Nombre común: Rocoto

\section{Materiales, equipos e instrumentos analíticos de laboratorio}

Equipo de extracción por fluido supercrítico de $\mathrm{CO}_{2}\left(\mathrm{SFE}-\mathrm{CO}_{2}\right)$ marca THAR Instruments SFE - 100 - 2 - FMC. Espectrofotómetro UVI visible INC- Medilab; instrumento analítico de Cromatografía Líquida de Alta Eficiencia (HPLC) marca Shimadzu, con columna cromatográfica Ultra C18, $250 \mathrm{~mm}, 4.6 \mathrm{~mm}$ ID x 5 um, Temperatura límite $80^{\circ} \mathrm{C}$

\section{Insumos y reactivos}

$\mathrm{CO}_{2}$ de $99,999 \%$ de pureza con certificación de PRAXAIR, patrones de capsaicinoides de Sigma Aldrich y carotenoides de carotenature; acetonitrilo, hexano, metanol, etanol, agua de grado HPLC.

\section{Acondicionamiento de la materia prima}

Se realizó el secado de la pulpa y placenta del
Capsicum sp, por convección de aire caliente y se determinó el tamaño de partícula de Capsicum pubescens molido. Método granulométrico Test Sieving (Nagy \& Simandi, 2008), también se determinó del porcentaje de humedad. Método gravimétrico (FAO. 2004).

Procesos de extracción de capsaicinoides y carotenoides por fluido supercrítico, se realizó por el Método Yao et. al., (1994) y Zao (2006).

\section{Análisis de capsaicinoides y carotenoides}

Se realizó la determinación del contenido total de capsaicinoides y carotenoides. Método espectrofotométrico, ISO 7543 - 1 y AOAC (970.64) y las fracciones predominantes de capsaicinoides por HPLC. Método AOAC (995.03), método Hoffman (1983).

\section{Diseño experimental estadístico}

Para probar los tratamientos del proceso de extracción por dióxido de carbono supercrítico se utilizó un diseño completamente aleatorio, con arreglo factorial 22, con un nivel de confianza del $5 \%$, ANOVA y prueba de comparación múltiple de Duncan. El procesamiento de datos se realizó a través del Statistical Analysis System (SAS) V® 9.1 .

\section{RESULTADOS Y DISCUSIÓN}

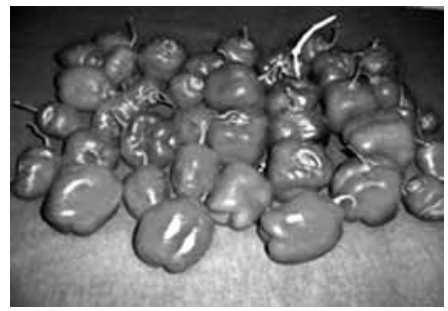

Figura 1. Proceso de extracción de Capsaicinoides de Capsicum sp. por dióxido de carbono supecrítico 
Según el diseño experimental planteado con 4 tratamientos, se realizó la extracción de la oleorresina de rocoto, el cual fue acondicionado a una humedad de 13 a $14 \%$ por secado con aire caliente y secado a vacio a $40^{\circ} \mathrm{C}$ (Figura 2), luego fue molido y tamizado a un tamaño de partículas entre 425 u a $1 \mathrm{~mm}$. Para permitir una adecuada superficie de exposición de la muestra con el $\mathrm{CO}_{2}$ supercrítico. Para cada tratamiento se utilizó aproximadamente $28 \mathrm{~g}$ de muestra seca. En el cuadro 1, se muestra los resultados de la cantidad de oleorresina extraída, en condiciones de presión (bar): 200 (-1) y 400 (1), y temperatura $\left({ }^{\circ} \mathrm{C}\right): 40(-1)$ y $60(1)$.

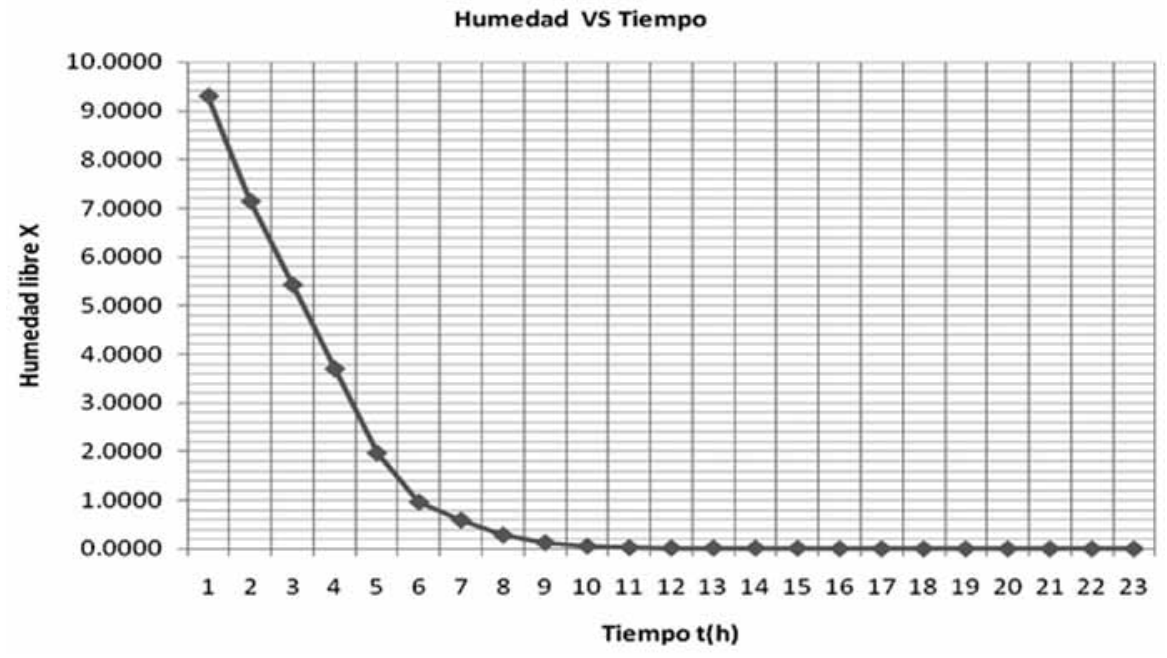

Figura 2. Pérdida de humedad del pericarpio de rocoto durante el tiempo de secado

Cuadro № 1. Cantidad de oleorresina total de Capsicum sp. (rocoto)

\begin{tabular}{cccrcccc}
\hline Tratamiento & $\begin{array}{c}\text { Presión } \\
(\mathrm{b} \text { bar })\end{array}$ & $\begin{array}{c}\text { Temperatura Muestra } \\
\left({ }^{\circ} \mathrm{C}\right)\end{array}$ & $\begin{array}{c}\text { Humedad } \\
\text { seca }(\mathrm{g})\end{array}$ & $\begin{array}{c}\text { Oleorresina } \\
(\%)\end{array}$ & $\begin{array}{c}\text { Rendimiento } \\
(\mathrm{g})\end{array}$ & $(\%)$ \\
\hline $\mathrm{T}_{1}$ & -1 & -1 & 28.450 & $14.70 \%$ & 0.319 & 1.121 \\
$\mathrm{~T}_{2}$ & -1 & 1 & 28.938 & $14.90 \%$ & 0.500 & 1.728 \\
$\mathrm{~T}_{3}$ & 1 & -1 & 28.546 & $13.20 \%$ & 0.146 & 0.511 \\
$\mathrm{~T}_{4}$ & 1 & 1 & 28.529 & $13.60 \%$ & 0.154 & 0.540 \\
\hline
\end{tabular}

El tratamiento dos (T2) a presión de 200 bar y temperatura de $60{ }^{\circ} \mathrm{C}$ permitió obtener la mayor cantidad de oleorresina, seguido por el tratamiento uno (T1), comparado con los tratamientos tres y cuatro, los cuales reportan una menor cantidad de oleorresina. Los rendimientos totales de oleorresina y sus componentes de capsaicinoides de rocoto van a depender de muchos factores, como del pretratamiento de la matriz vegetal, así como del porcentaje de humedad final de la muestra seca y del tamaño de partícula adecuada. Del Valle et al., (2003) señala que las muestras molidas a partir de laminados y luego peletizadas y acondicionadas a baja humedad tienen un rendimiento mayor que aquellas no molidas previamente. Asimismo, la presión y la temperatura afectan la solubilidad de la oleorresina en $\mathrm{CO}_{2}$, aunque ello también 
depende de la materia prima. En general, la temperatura de $60{ }^{\circ} \mathrm{C}$ presenta una mayor selectividad en la extracción con $\mathrm{CO}_{2}$ (JarénGalán et al., 1999). Aunque la mayoría de los investigadores recomiendan $40{ }^{\circ} \mathrm{C}$ en vez de 55 ${ }^{\circ} \mathrm{C}$; Daood et al. (2002) obtuvieron un máximo de rendimiento de extracción $(\sim 11,5 \%)$ a 40 MPa y 55 ${ }^{\circ} \mathrm{C}$. Una mínima densidad del $\mathrm{CO}_{2}$ es necesaria para extraer oleorresina picante (entre 600-1000 $\mathrm{kg} / \mathrm{m} 3)$. Una mayor densidad también aumenta la economía de la Extracción Supercrítica de $\mathrm{CO}_{2}$ con independencia de la temperatura utilizada, porque al igual que con un aumento de Presión, aumenta la solubilidad del aceite en $\mathrm{CO}_{2}$ (Daood et al., 2002).

\section{Determinación de las fracciones predominantes de capsaicinoides de rocoto}

Los cromatogramas de rocoto $(A)$ y patrón $(B)$ (Figura 3), obtenidos, permitieron identificar y cuantificar las fracciones de capsaicinoides: Nordihidrocapsaicina (NDHC), Capsaicina (CAP) y Dihidrocapsaicina (DHC). En el cual se observa dos picos desconocidos (a y b) y en el cuadro 2, se observa el contenido de cada fracción a diferentes tratamientos de extracción SF-CO ${ }_{2}$. El tratamiento $\mathrm{T} 2$ a $60^{\circ} \mathrm{C}$ y 200 bar, permitió la mayor extracción a diferencia de las otras condiciones, siendo NDHC $=770.51$ $(17 \%) ; \mathrm{CAP}=1471.22(33 \%)$ y $\mathrm{DHC}=2116.19$ $(49 \%)$, haciendo un total de capsaicinoides de $4357.92 \mathrm{ug} / \mathrm{g} \mathrm{ms}$, y con una pungencia de $\mathrm{SHU}=65368.76$; la relación de estas fracciones 1:0.69:0.36 muestra que la dihidrocapsaicina se encuentra en mayor proporción en esta especie de Capsicum pubescen (rocoto). Estos resultados fueron comparados con el cromatograma patrón de capsaicinoides comerciales y se observa que los tiempos de retención de las tres fracciones $\mathrm{NDHC}=7,7 ; \mathrm{CAP}=7,9$ y $\mathrm{DHC}=12,3 \mathrm{~min}$. Coinciden con las de rocoto. Asimismo, el nivel de unidades respuesta del integrador ( $m A U$ ) es mucho mayor en los picos de los capsaicinoides de rocoto, especialmente el de NDHC en comparación con el estándar. Las unidades de respuesta del integrador son una función directa de la concentración del compuesto identificado, a mayor mAU mayor es la concentración del compuesto extraído. La eficiencia de la mayor extracción de los capsaicinoides por efecto de la temperatura y presión del $\mathrm{CO}_{2}$ supercrítico, puede deberse al aumento del proceso de transferencia de masa dentro del tejido seco del vegetal, lo cual se traduce en el incremento de la permeabilidad de la membrana citoplasmática (Butz et al, 2003; Antonio y Gonzalo, 2005; Ahmed y Ramaswamy, 2006).

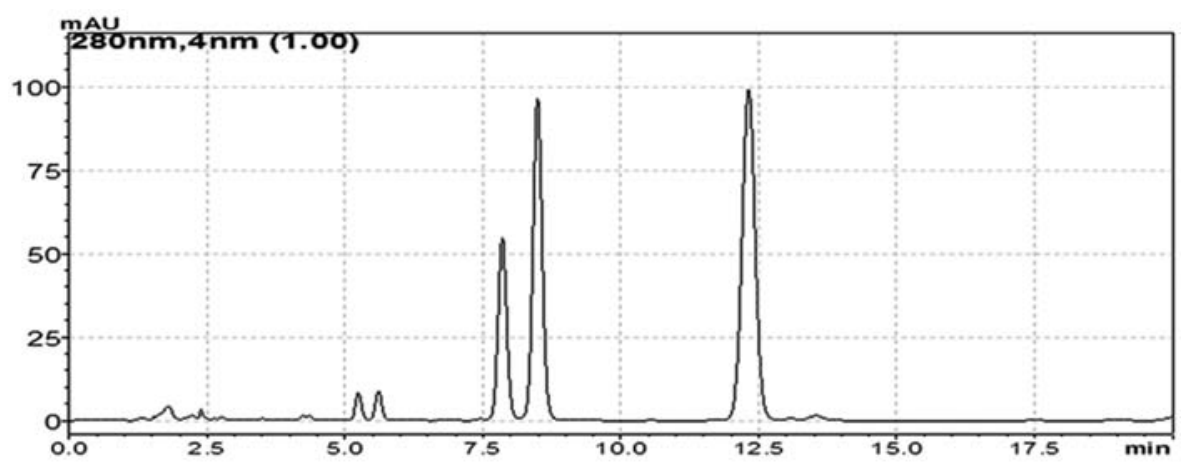

Figura $\mathrm{N}^{\circ}$ 3. Cromatogramas de fracciones de Capsaicinoides extraído por fluido supercrítico de CO2: a y b) desconocidos, Nordihidrocapsaicina (NDHC), Capsaicina (CAP), Dihidrocapsaicina (DHC). 
Peusch et al (1997), señala que en la extracción las fracciones 10 y $9 \%$ más alto de NDHC y DHC por SFE a $80^{\circ} \mathrm{C}$ de Chile de USA, obtuvo 760 y $20 \%$ menos de CAP, respectivamente. Estas $\mathrm{ug} / \mathrm{g}$ de capsaicinoides y fracciones de 53,40 y variaciones probablemente se deben al tipo de $7 \%$ de CAP, DHC y NDHC, respectivamente. Sin especie, cultivares inter e intra especies, suelo, embargo en el estudio realizado obtuvimos 5,7 lugar de cultivo y manejo agrícola.

veces más de capsaicinoides totales, respecto a

\begin{tabular}{ccccccc}
\hline $\begin{array}{c}\text { Extracción } \\
\text { SF- } \\
\mathrm{CO}_{2}\end{array}$ & $\begin{array}{c}\text { NDHC } \\
\text { (ug/g } \\
\text { ms) }\end{array}$ & $\begin{array}{c}\text { CPS } \\
\text { (ug/g } \\
\text { ms) }\end{array}$ & $\begin{array}{c}\text { DHC } \\
\text { (ug/g ms) }\end{array}$ & $\begin{array}{c}\text { Capsaicinoides } \\
\text { Totales } \\
\text { (ug/g ms) }\end{array}$ & $\begin{array}{c}\text { SHU } \\
\text { (ug/g } \\
\text { ms) }\end{array}$ & DHC:CPS:NDHC \\
\hline & & & & & & \\
T1 & 214.46 & 400.62 & 577.04 & 1192.11 & 17881.62 & $1: 0.69: 0.37$ \\
& 770.51 & 1471.22 & 2116.19 & 4357.92 & 65368.76 & $1: 0.69: 0.36$ \\
T3 & 445.31 & 937.98 & 1239.42 & 2622.71 & 39340.58 & $1: 0.75: 0.35$ \\
T4 & 18.91 & 39.95 & 43.52 & 102.38 & 1535.73 & $1: 0.91: 0.43$
\end{tabular}

Cuadro 2. Contenido de Nordihidrocapsaicina (NDHC), Capsaicina (CAP), Dihidrocapsaicina (DHC), capsaicinoides totales, unidades Scoville (SHU) y relación DHC:CPS:NDHC de rocoto, extraído por SF-CO2.

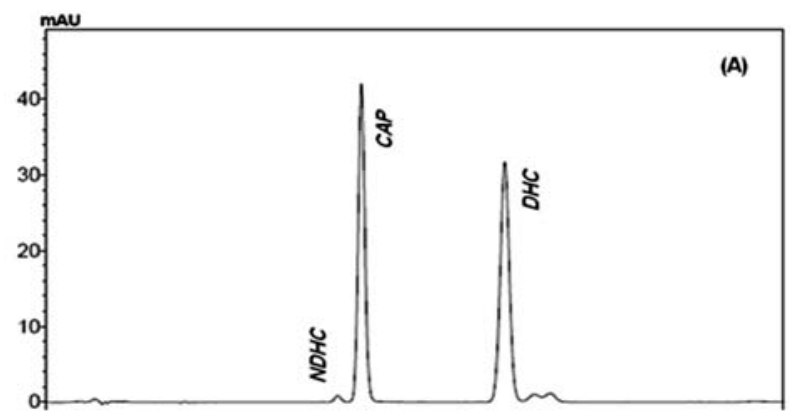

En Capsicum annuum la proporción es cercana a 1:1, en tanto que para $C$. frutescens $L$. es 2:1 (Manirakiza et al. (2003); además, Cruz (2007) observó un mayor contenido de DH en muestras de $\mathrm{C}$. pubescens. Esta última especie corrobora a los resultados obtenidos de Capsicum sp (rocoto).

En conclusión, los resultados de este estudio demostraron que el SFE-CO2 es un método disponible para extracciones selectivas de capsaicinoides de Capsicum seco y molido con $14 \%$ de humedad. La ventaja de este método comparado con otros métodos, requiere poca preparación de la materia prima, corto tiempo de extracción y requiere poca preparación para los análisis de HPLC y hay muy pocas señales de interferencia en los cromatogramas, lo cual facilita la integración del contenido de capsaicinoides y cuantificar su concentración total, fraccionada, y determinar las unidades de pungencia en unidades SCOVILLE. Esta valorización química de capsaicinoides de rocoto sirve de orientación científica y tecnológica, que podría traducirse en términos económicos por su contenido de compuestos bioactivos, que lo hace un excelente recurso natural promisorio y que merece mejorar su cultivo y producción. 
Extracción por fluido supercrítico de $\mathrm{CO}_{2}$ y cuantificación de capsaicinoides de rocoto (Capsicum Pubescen) del Valle del Mantaro

\section{REFERENCIAS BIBLIOGRÁFICAS}

Antonio T. J.; Gonzalo V. G. Commercial opportunities and research challenges in the high pressure processing of foods. J. Foods Eng., 67, 95-112. 2005.

Ahmed J.; Ramswamy H. S. High pressure processing of fruits and vegetables. Stewart Postharvest Rev. 1 - 10. 2006.

Butz P.; Fernández G. A.; Lindauer R; Dieterich S; Bognar A.; Tauscher B.Influence of ultra high pressure processing on fruit and vegetable products. J. Food Eng. 56, 233-236. 2003.

Cruz P. A. B; González H. M. A; Gutiérrez E. A. A.; Gardea B. M.; Pérez G. Capsaicinoides, vitamina $C$ y heterosis durante el desarrollo del fruto de chile manzano. Agrociencia 41: 627-635. 2007.

Daood HG; Ille's V; Gnayfeed MH; Mészáros B; Horvath G.; Biacs PA. Extraction of pungent spice paprika by supercritical carbon dioxide and subcritica propane. J. Superc. Fluids, 23,143152. 2002.

Del Valle JM.; Jimenez M.; De la Fuente JC. Extraction kinetics of pre-pelletized Jalapeño peppers with supercritical CO2. J Superc. Fluids, 25, 33-44. 2003. Hoffman P; \& Lego, M. G. Separation and -1305.1994.

Quantification of red pepper major heat
Norma Gamarra Mendoza, Ivan Bontemps Hernández, Edith Coronel Bonifacio, Silvia Velásquez Rodríguez y Servan Granza Muñoz

principles by reverse - phase high pressure liquid chromatography. J. Agric. Food Chem; 31, 1326 - 1330. 1983.

Jarén Galán M.; Nienaber U.; \& Schwartz S. J. Paprika (Capsicum annuum) oleoresin extraction with supercritical carbon dioxide. J. Agric. Food Chem; 47, 3558-3564. 1999.

Nagy B. Effects of particle size distribution, moisture content, and initial oil content on the supercritical fluid extraction of paprika. J. Supercritical Fluid , 46, 293 - 298. 2008.

Nagy, B; Simandi B. Effects of particle size distribution, moisture content, and initial oil content on the supercritical fluid extraction of paprika. J. Supercritcal Fluid , 46, 293 - 298. 2008.

Salgado R. M; Botello A. E; Rico M. R.; Jimenez I. H.; Cardenas M. M.; Navarrete B. J. Enzymatic Treatment To improve Extraction of Capsaicinoids and Carotenoids from Chili (Capasicum annuum) Fruits. J. Agric. Food Chem; 56, 10012 - 10018. 2006.

Yao J; Muraleedharan G. Supercritical Carbon dioxide Extraccion from Scoth Bonnet (C. annumm) and Quantification of capsaicin and dihydrocapsaicin. J. Agric. Food Chem; 42, 1303 